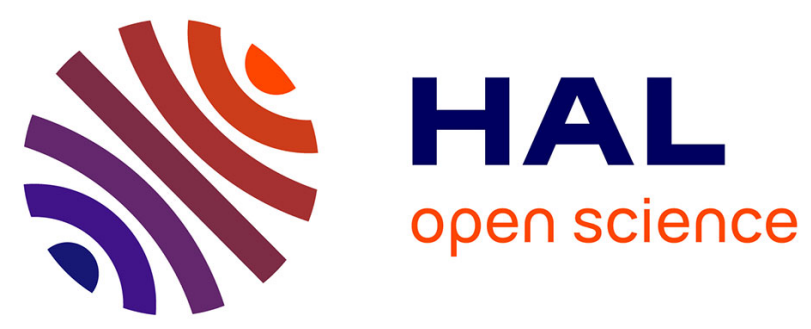

\title{
Convergence rate of individual and global quantities in direct numerical simulations
}

Cédric Flageul, Iztok Tiselj

\section{To cite this version:}

Cédric Flageul, Iztok Tiselj. Convergence rate of individual and global quantities in direct numerical simulations. Physics of Fluids, 2018, 30 (11), 10.1063/1.5052696 . hal-01929582

\section{HAL Id: hal-01929582 \\ https://hal.science/hal-01929582}

Submitted on 21 Nov 2018

HAL is a multi-disciplinary open access archive for the deposit and dissemination of scientific research documents, whether they are published or not. The documents may come from teaching and research institutions in France or abroad, or from public or private research centers.
L'archive ouverte pluridisciplinaire $\mathbf{H A L}$, est destinée au dépôt et à la diffusion de documents scientifiques de niveau recherche, publiés ou non, émanant des établissements d'enseignement et de recherche français ou étrangers, des laboratoires publics ou privés. 


\title{
Convergence rate of individual and global quantities in Direct Numerical Simulations
}

\author{
Cédric Flageul ${ }^{1, a)}$ and Iztok Tiselj ${ }^{1, b}$ ) \\ Reactor engineering division, Jožef Stefan institute, Ljubljana, Slovenia
}

(Dated: 6 November 2018)

Typical individual quantities in Direct Numerical Simulations of statistically steady flows are converging at a rate of $1 / \sqrt{T}$, where $T$ is the averaging time of the simulation. However, global quantities that represent integral momentum balance in the computational domain can exhibit a faster convergence rate of $1 / T$. This faster convergence rate is analysed and explained. Theoretical predictions are supported with a Direct Numerical Simulation.

Keywords: Direct Numerical Simulation, Uncertainty Quantification, Sampling Error

As the existing computational power available increases, Direct Numerical Simulation (DNS) establishes itself as one of the most accurate way to analyse fluid flows. Thanks to Oliver et al! $\stackrel{1}{\text {, }}$, practitioners are now able to estimate the statistical uncertainty plaguing their results. This is a major step towards uncertainty quantification as the statistical uncertainty is usually the main source of error in DNS.

Recently, Thompson et al. ${ }^{[}$proposed and applied a methodology to evaluate the statistical error plaguing the averaged velocity and the Reynolds stress in plane channel flows. This methodology was used by Andrade et al. ${ }^{[}$. Their Figure 6 suggested a residual norm decreasing as $\frac{1}{T}$, with $T$ the averaging time.

However, following Oliver et al $\stackrel{1}{1}$, it is known that individual statistical quantities are converging slower, at a rate of $\frac{1}{\sqrt{T}}$. This is the expected convergence rate for an uncorrelated signal, and for a weakly correlated one approaching independence at large separations, such as a turbulent signal (Theorem 27.4 in Billingsley ${ }^{4}$ ).

In the present paper, we show that both aforementioned convergence rates hold and are not contradictory. This is confirmed by statistics taken from a DNS. Thus, we demonstrate that the methodology for evaluation of statistical uncertainties proposed by Thompson et al. ${ }^{[2}$ is not conservative as it provides an error which is converging faster than individual quantities.

The structure of the remaining part of the paper is as follows. First, we show that the residual norms proposed by Thompson et a ${ }^{2}$ are converging at a rate of $\frac{1}{T}$. Then, we show DNS results in agreement with our analysis, alongside with some concluding remarks and comments.
The following is tailored to the time scheme used by Andrade et al ${ }^{[3}$. It could be simply adapted to fit another time scheme. The velocity in direction $i$ and at time step $n$ is written $u_{i}^{n}$. The pressure at time step $n$ is written $p^{n}$. The time step is written $d t$. Spatial derivativation in direction $i$ is written $\partial_{i}$ and $\delta_{i, j}$ is the Kronecker delta. In the present study, $X, Y$ and $Z$ stand for the streamwise, wall-normal and spanwise directions, respectively. The prediction step is a combination of a $2^{\text {nd }}$ order Adams-Bashforth and Crank-Nicolson, and there is an imposed constant source term $(f)$ in the streamwise direction driving the flow:

$$
\begin{aligned}
\frac{u_{i}^{*}-u_{i}^{n}}{d t} & =-\frac{3}{2} \partial_{j}\left(u_{i}^{n} u_{j}^{n}\right)+\frac{1}{2} \partial_{j}\left(u_{i}^{n-1} u_{j}^{n-1}\right)-\partial_{i} p^{n} \\
& +\frac{1}{R e} \partial_{j j}\left(\frac{u_{i}^{*}+u_{i}^{n}}{2}\right)-f \delta_{i X}
\end{aligned}
$$

The correction step enforces the continuity equation

$$
\frac{u_{i}^{n+1}-u_{i}^{*}}{d t}=-\partial_{i} p^{n+1}+\partial_{i} p^{n}
$$

as the pressure $p^{n+1}$ is obtained with the following Poisson equation:

$$
\partial_{i i} p^{n+1}=\partial_{i i} p^{n}+\frac{\partial_{i} u_{i}^{*}}{d t}
$$

From equation (2), one can derive

$$
u_{i}^{*}=u_{i}^{n+1}+d t\left(\partial_{i} p^{n+1}-\partial_{i} p^{n}\right)
$$

Equation (4) allows us to eliminate $u_{i}^{*}$. The resulting time scheme is:

$$
\frac{u_{i}^{n+1}-u_{i}^{n}}{d t}=-\frac{3}{2} \partial_{j}\left(u_{i}^{n} u_{j}^{n}\right)+\frac{1}{2} \partial_{j}\left(u_{i}^{n-1} u_{j}^{n-1}\right)-\partial_{i} p^{n+1}+\frac{1}{R e} \partial_{j j}\left(\frac{u_{i}^{n+1}+u_{i}^{n}}{2}+\frac{d t}{2} \partial_{i}\left(p^{n+1}-p^{n}\right)\right)-f \delta_{i X}
$$

At this point, the time-discrete momentum equation (5) is averaged in time. This corresponds to a summation
b)Electronic mail: iztok.tiselj@ijs.si 
from the time step $n=1$ to $n=N$, followed by a division by $N$ :

$$
\begin{aligned}
& \frac{u_{i}^{N+1}-u_{i}^{1}}{N d t}+\frac{1}{2 N} \partial_{j}\left(u_{i}^{N} u_{j}^{N}-u_{i}^{0} u_{j}^{0}\right)-\frac{1}{N R e} \partial_{j j}\left(\frac{u_{i}^{N+1}-u_{i}^{1}}{2}+\frac{d t}{2} \partial_{i}\left(p^{N+1}-p^{1}\right)\right)+\frac{\partial_{i} p^{N+1}-\partial_{i} p^{1}}{N} \\
= & \frac{\sum_{k=1}^{N}-\partial_{j}\left(u_{i}^{k} u_{j}^{k}\right)-\partial_{i}\left(p^{k}\right)+\frac{1}{R e} \partial_{j j}\left(u_{i}^{k}\right)-f \delta_{i, X}}{N}
\end{aligned}
$$

On the right-hand side of equation (6) is the exact momentum equation averaged in time over the simulation. Thus, the left-hand side of this equation corresponds to a residual. When $N$ is large enough, the velocity and pressure fields at the initial time steps are independent from the ones at the final time steps. As the flow is statistically steady, the initial and final fields are described by the same probability density function. Therefore, the collected statistics will converge towards the exact time-averaged momentum equation at a rate of $\frac{1}{N}$, or $\frac{1}{T}$. This convergence rate was obtained without any specific assumption. Thus, it does not depends on the geometry or on the Reynolds number, and is valid everywhere inside the computational domain, as long as the initial turbulent flow is fully developped.

Hereafter, we focus on the streamwise and spanwise momentum equations which are useful as indicators of the statistical uncertainty in the channel flow. Both equations are averaged over the homogeneous directions $X$ and $Z$. Quantities averaged in space are written with one overbar. The resulting space-averaged time-averaged discrete momentum equations are:

$$
\begin{aligned}
& \frac{\overline{u_{X}^{N+1}}-\overline{u_{X}^{1}}}{N d t}+\frac{1}{2 N} \partial_{Y}\left(\overline{u_{X}^{N} u_{Y}^{N}}-\overline{u_{X}^{0} u_{Y}^{0}}\right)-\frac{1}{N R e} \partial_{Y Y}\left(\frac{\overline{u_{X}^{N+1}}-\overline{u_{X}^{1}}}{2}\right)=\frac{\sum_{k=1}^{N}-\partial_{Y}\left(\overline{u_{X}^{k} u_{Y}^{k}}\right)+\frac{1}{R e} \partial_{Y Y}\left(\overline{u_{X}^{k}}\right)-f}{N}(7) \\
& \frac{\overline{u_{Z}^{N+1}}-\overline{u_{Z}^{1}}}{N d t}+\frac{1}{2 N} \partial_{Y}\left(\overline{u_{Z}^{N} u_{Y}^{N}}-\overline{u_{Z}^{0} u_{Y}^{0}}\right)-\frac{1}{N R e} \partial_{Y Y}\left(\frac{\overline{u_{Z}^{N+1}}-\overline{u_{Z}^{1}}}{2}\right)=\frac{\sum_{k=1}^{N}-\partial_{Y}\left(\overline{u_{Z}^{k} u_{Y}^{k}}\right)+\frac{1}{R e} \partial_{Y Y}\left(\overline{u_{Z}^{k}}\right)}{N}
\end{aligned}
$$

To measure the convergence rate, we define the following quantities:

$$
\begin{aligned}
E_{0}^{X}(y) & =\frac{\sum_{k=1}^{N}-\partial_{Y}\left(\overline{u_{X}^{k} u_{Y}^{k}}\right)+\frac{1}{R e} \partial_{Y Y}\left(\overline{u_{X}^{k}}\right)-f}{N} \\
E_{0}^{Z}(y) & =\frac{\sum_{k=1}^{N}-\partial_{Y}\left(\overline{u_{Z}^{k} u_{Y}^{k}}\right)+\frac{1}{R e} \partial_{Y Y}\left(\overline{u_{Z}^{k}}\right)}{N} \\
\left\|E_{0}^{i}\right\|^{2} & =\frac{1}{R e_{\tau}} \int_{y^{+}=0}^{R e_{\tau}} E_{0}^{i}\left(y^{+}\right)^{2}
\end{aligned}
$$

Based on this, one can recover the residual error $E_{R}^{i}$, as defined by Andrade et al $\stackrel{3}{[}$ :

$$
\begin{aligned}
E_{R}^{i}\left(y^{+}\right) & =\int_{l=0}^{y^{+}} E_{0}^{i}(l) \\
\left\|E_{R}^{i}\right\|^{2} & =\frac{1}{R e_{\tau}} \int_{y^{+}=0}^{R e_{\tau}} E_{R}^{i}\left(y^{+}\right)^{2}
\end{aligned}
$$

The residual error $E_{U}^{i}$, also defined by Andrade et al ${ }^{[3}$, can be obtained by integrating $E_{R}^{i}$. Obviously, $\left\|E_{0}^{i}\right\|$, $\left\|E_{R}^{i}\right\|$ and $\left\|E_{U}^{i}\right\|$ should have the same convergence rate.

For the spanwise momentum equation, the global quantity $\left\|E_{0}^{Z}\right\|^{2}$ is completed by the following individual quantities:

$$
\begin{gathered}
Z-\text { Conv }=\frac{1}{R e_{\tau}} \int_{y^{+}=0}^{R e_{\tau}}\left(\frac{\sum_{k=1}^{N} \partial_{Y}\left(\overline{u_{Z}^{k} u_{Y}^{k}}\right)}{N}\right)^{2} \\
Z-\operatorname{Diff}=\frac{1}{R e_{\tau}} \int_{y^{+}=0}^{R e_{\tau}}\left(\frac{\sum_{k=1}^{N} \frac{1}{R e} \partial_{Y Y}\left(\overline{u_{Z}^{k}}\right)}{N}\right)^{2}
\end{gathered}
$$

Regarding the conversion towards wall-units, a friction velocity - the theoretical one — can be derived from the constant source term $f$ used in the momentum equation. Another friction velocity - the measured one - can be derived from the averaged streamwise velocity. In the present study, the former is used as it is not plagued by any statistical uncertainty. The definition of $E_{R}^{i}$, as given by equation 10$]$, uses an integration constant to enforce $E_{R}^{i}\left(y^{+}=0\right)=0$. In the present study, we weakly enforce this condition at $y^{+}=0$ by using an integration constant derived from the theoretical friction velocity, as in Andrade et al $\stackrel{3}{3}$. This choice, combined with the symmetry of the case at hand, strongly enforces $E_{R}^{i}\left(y^{+}=R e_{\tau}\right)=0$.

Hereafter, we present DNS results supporting our analysis. For convenience, all quantities plotted are integrated squared residuals. Thus, expected convergence rates are also squared and individual quantities are expected to converge at a rate of $\frac{1}{T}$, while global ones such as $\left\|E_{0}^{i}\right\|^{2}$ and $\left\|E_{R}^{i}\right\|^{2}$ are expected to converge at a rate of $\frac{1}{T^{2}}$. 


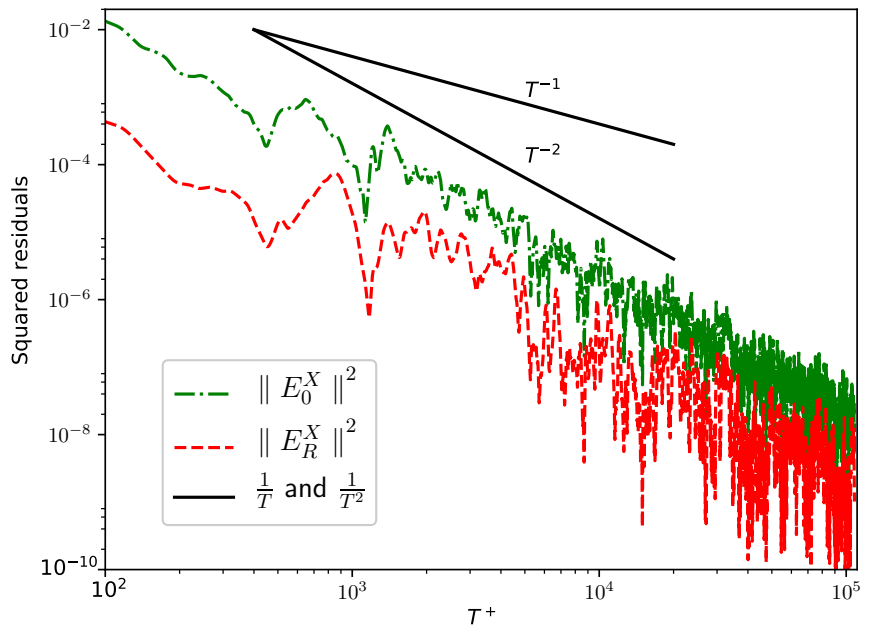

FIG. 1. Convergence of global quantities associated with the streamwise momentum as a function of the averaging time. Dotted green line: $\left\|E_{0}^{X}\right\|^{2}$. Dashed red line: $\left\|E_{R}^{X}\right\|^{2}$. Black lines: Convergence rates of $\frac{1}{T}$ and $\frac{1}{T^{2}}$.

The present DNS was performed with the pseudospectral code previously used in Flageul and Tisel $5^{5}$. The friction Reynolds number is 180 . The shape and the resolution of the computation is equal to the DNS of Moser et. al! $\left[L_{X}=4 \pi h, L_{Y}=2 h, L_{Z}=\frac{4 \pi h}{3}\right], \Delta_{X}^{+}=17.7$, $0.054 \leq \Delta_{Y}^{+} \leq 4.4$ and $\Delta_{Z}^{+}=5.90$. This resolution was recognized as slightly under-resolved DNS in the study by Vreman and Kuerten ${ }^{7}$. This is not really an issue here as this paper is focused only on statistical uncertainty and not on the numerical accuracy. The time step in the DNS is 0.027 wall-units, and statistics are collected every 10 time steps. 4 million time steps are performed, so the averaging time is 108000 wall-units and the effective averaging time, as proposed by Vinuesa et. al $\frac{8}{\text {, }}$, is $T^{\star}=\frac{T u_{\tau}}{h} \frac{L_{X} L_{Z}}{L_{X, \min } L_{Z, \min }}=1760$, with $L_{X, \min }=6 h$ and $L_{Z, \min }=3 h$.

As shown in Figure 1, the global quantities $\left\|E_{0}^{X}\right\|^{2}$ and $\left\|E_{R}^{X}\right\|^{2}$ associated with the streamwise momentum equation are converging at the expected rate of $\frac{1}{T^{2}}$.

As shown in Figure 2, the squared residuals associated with individual quantities are converging at a rate of $\frac{1}{T}$, while the squared residual associated with the global quantity $\left\|E_{0}^{Z}\right\|^{2}$ is converging at the faster rate of $\frac{1}{T^{2}}$.

For the sake of completeness, regressions were performed to extract the convergence rate of the quantities plotted in Figures 1 and 2, The regression was restricted to averaging times longer than $10^{4}$ samples $-10^{5}$ time steps, or 2700 time units - to limit transient effects. Table [ shows that the convergence rate of global quantities is very close to the theoretical prediction. Table $\Pi$ shows that the individual quantities are also converging at a rate very close to the theoretical prediction (Oliver et al ${ }^{1}$, Billingsley $\left.{ }^{4}\right)$.

The authors have presented an original analysis show-

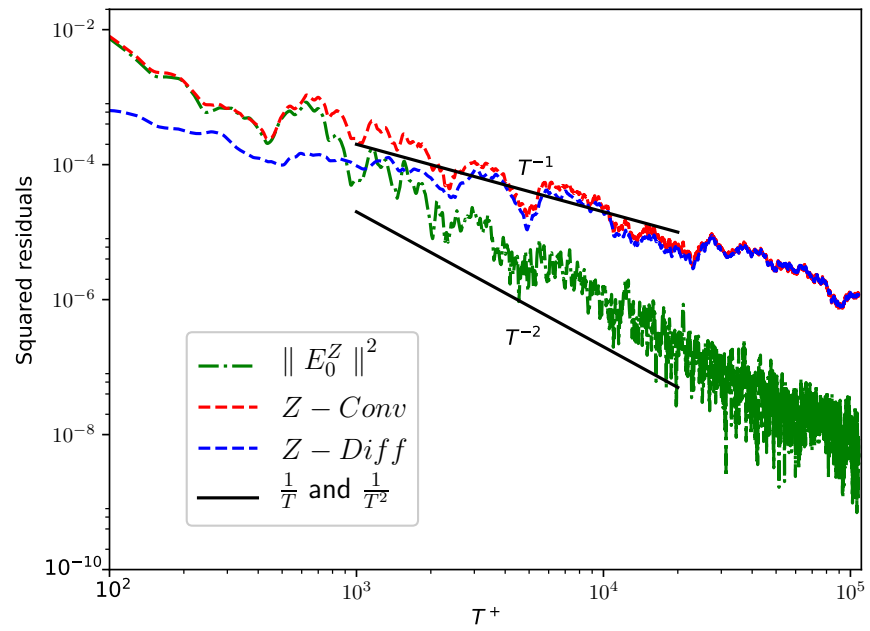

FIG. 2. Convergence of individual and global quantities associated with the spanwise momentum as a function of the averaging time. Dotted green line: $\left\|E_{0}^{Z}\right\|^{2}$. Dashed red line: $Z-C o n v$. Dashed blue line: $Z-\operatorname{diff}$. Black lines: Convergence rates of $\frac{1}{T}$ and $\frac{1}{T^{2}}$.

TABLE I. Convergence rate for global quantities, averaging time $T^{+}>2700$.

\begin{tabular}{ccccc}
\hline \hline & $\left\|E_{0}^{X}\right\|^{2}$ & $\left\|E_{0}^{Z}\right\|^{2}$ & $\left\|E_{R}^{X}\right\|^{2}$ & $\left\|E_{R}^{Z}\right\|^{2}$ \\
\hline Convergence Rate & $T^{-1.98}$ & $T^{-2.05}$ & $T^{-1.87}$ & $T^{-2.11}$ \\
\hline \hline
\end{tabular}

ing that different quantities can have different convergence rates, and have provided DNS evidences supporting it. Overall, individual quantities are expected to converge at a rate of $\frac{1}{\sqrt{T}}$. However, a few global quantities, directly related to the integral equations solved are converging faster, at a rate of $\frac{1}{T}$. The only global quantities the authors could identify are the ones directly derived from the conservation equations solved, as expressed equation (6).

One could try to multiply equation (5) by $\left(u_{i}^{n+1}+u_{i}^{n}\right)$ to obtain an equation for the kinetic energy as a global quantity. However, summation over time steps no longer leads to simplification. Thus, it seems that no other global quantity converging at a rate of $\frac{1}{T}$ can be derived on this ground, except linear transformations of the previous ones. As integration and derivation are linear transformations, the time-averaged equation of conservation of vorticity is also a global quantity converging at a rate of $\frac{1}{T}$.

TABLE II. Convergence rate for individual quantities, averaging time $T^{+}>2700$.

\begin{tabular}{lcc}
\hline \hline & $Z-$ Conv & $Z-\operatorname{diff}$ \\
\hline Convergence Rate & $T^{-1.22}$ & $T^{-1.11}$ \\
\hline \hline
\end{tabular}


The present analysis was focused on the momentum equation, but will also apply to transported scalars, such as the temperature or the enthalpy. In addition, this analysis is not restricted in any way to simple geometries such as the channel flow studied here and can be directly applied to DNS of more complex geometries. The main requirement is that the case studied must be statistically steady. The other is that the DNS should rely on a classical combination of implicit and explicit time schemes. The presented analysis should also apply to time schemes with a variable time step.

As a side remark, our analysis shows that the residuals associated with the global momentum equation depend only on the initial and final fields. Thus, provided one has kept those fields, one can estimate the error on the averaged momentum equation without using any averaged quantity. Indeed, this is of very limited use, except for an efficient on-the-fly estimation of this global error during the simulation.

A potentially interesting error estimator regarding individual statistical quantities for cases with symmetry or invariance under rotation could be derived from the lack of symmetry or invariance in the measured profile. This is left for future prospects. As a side note, the authors would like to stress that convergence rates correspond to asymptotic limits and long averaging time can be necessary to observe them, especially for low probability events (Bauer et al. ${ }^{-}$).

The authors would like to thank the Slovenian Re- search Agency for funding the study under the research project P2-0026. Data associated with the present paper are available online at https://repo.ijs.si/CFLAG/ convergence_rate under the GNU GPL v3 licence.

${ }^{1}$ T. A. Oliver, N. Malaya, R. Ulerich, and R. D. Moser, "Estimating uncertainties in statistics computed from direct numerical simulation," Physics of Fluids 26, 035101 (2014).

${ }^{2}$ R. L. Thompson, L. E. B. Sampaio, F. A. de Bragança Alves, L. Thais, and G. Mompean, "A methodology to evaluate statistical errors in DNS data of plane channel flows," Computers \& Fluids 130, 1-7 (2016)

${ }^{3}$ J. R. Andrade, R. S. Martins, R. L. Thompson, G. Mompean, and A. da Silveira Neto, "Analysis of uncertainties and convergence of the statistical quantities in turbulent wall-bounded flows by means of a physically based criterion," Physics of Fluids 30, 045106 (2018)

${ }^{4}$ P. Billingsley, Probability and measure (John Wiley \& Sons, 2008).

${ }^{5}$ C. Flageul and I. Tiselj, "Impact of unresolved smaller scales on the scalar dissipation rate in direct numerical simulations of wall bounded flows," International Journal of Heat and Fluid Flow 68, 173-179 (2017)

${ }^{6}$ R. D. Moser, J. Kim, and N. N. Mansour, "Direct numerical simulation of turbulent channel flow up to $R e_{\tau}=590$," Physics of fluids 11, 943-945 (1999)

'A. Vreman and J. G. Kuerten, "Comparison of direct numerical simulation databases of turbulent channel flow at $R e_{\tau}=180$," Physics of Fluids 26, 015102 (2014).

${ }^{8}$ R. Vinuesa, C. Prus, P. Schlatter, and H. M. Nagib, "Convergence of numerical simulations of turbulent wall-bounded flows and mean cross-flow structure of rectangular ducts," Meccanica 51, 3025-3042 (2016)

${ }^{9}$ C. Bauer, D. Feldmann, and C. Wagner, "On the convergence and scaling of high-order statistical moments in turbulent pipe flow using direct numerical simulations," Physics of Fluids 29, 125105 (2017) 